\title{
Sonic hedgehog protein regulates fibroblast growth factor 8 expression in metanephric explant culture from BALB/c mice: Possible mechanisms associated with renal morphogenesis
}

\author{
XING CHEN, XIAO-MING HOU, YOU-FEI FAN, YU-TING JIN and YU-LIN WANG \\ Department of Pediatrics, Provincial Hospital Affiliated to Shandong University, Jinan, Shandong 250021, P.R. China
}

Received August 9, 2015; Accepted June 17, 2016

DOI: $10.3892 / \mathrm{mmr} .2016 .5614$

\begin{abstract}
The sonic hedgehog (SHH) morphogen regulates cell differentiation and controls a number of genes during renal morphogenesis. To date, the effects of $\mathrm{SHH}$ on fibroblast growth factors (Fgfs) in embryonic kidney development remain unclear. In the present study, explants of BALB/c mouse embryonic kidney tissues were used to investigate the role of exogenous SHH on Fgf8 and Fgf10 expression levels ex vivo. Ureteric bud branches and epithelial metanephric derivatives were used to determine the renal morphogenesis with Dolichos biflorus agglutinin or hematoxylin-eosin staining. mRNA expression levels were determined using reverse transcription-quantitative polymerase chain reaction, while the protein expression levels were examined using immunohistochemistry and western blot analysis. During the initial stages of metanephric development, low levels of SHH, Fgf8, and Fgf10 expression were observed, which were found to increase significantly during more advanced stages of metanephric development. In addition, exogenous SHH protein treatment increased the number of ureteric bud branches and enhanced the formation of nephrons. Exogenous SHH reduced the Fgf8 mRNA and protein expression levels, whereas cyclopamine (an SHH-smoothened receptor inhibitor) interfered with SHH-mediated downregulation of Fgf8 expression. By contrast, exogenous $\mathrm{SHH}$ protein was not found to modulate Fgf10 mRNA and protein expression levels. In conclusion, these results indicate that the modulatory effects of SHH on BALB/c mouse metanephric explant cultures may involve the regulation of Fgf8 expression but not Fgf10 expression, which provides evidence for the functional role of Fgf proteins in renal morphogenesis.
\end{abstract}

Correspondence to: Dr Yu-Lin Wang, Department of Pediatrics, Provincial Hospital Affiliated to Shandong University, 324 Jing 5 Road, Jinan, Shandong 250021, P.R. China

E-mail: sdmed_pediatrics@163.com

Key words: sonic hedgehog, fibroblast growth factor, cyclopamine, renal morphogenesis, mouse culture

\section{Introduction}

The permanent (also know as the metanephric) kidney begins to develop on embryonic day 11 (E11) in mice, and around E35 in humans (1). At this stage, the metanephric blastema induces the caudal portion of the Wolffian (or mesonephric) duct to evaginate. An epithelial tubule forms, which is known as the ureteric bud, and invades the adjacent metanephric mesenchyme. During development, the branching ureteric bud tips induce the surrounding nephron progenitors of the metanephric blastema to proliferate and differentiate into nephrons. Signaling between the ureteric tip and the surrounding metanephric mesenchyme regulates the development of structures within the kidney $(2,3)$. The absorption of the ureteric bud, a process termed renal branching morphogenesis, ultimately constitutes the mature collecting duct system and is regulated by this signaling pathway (3). In addition, signaling between these tissues promotes the transformation of the metanephric mesenchyme to form the epithelial components, including the glomerulus and the distal tubule, in a process known as nephrogenesis $(2,4)$.

The morphogen sonic hedgehog-smoothened (SHH-SMO) signaling pathway serves an important role during mammalian kidney development. Binding of the SHH ligand to its receptor, patched (PTC), activates the transmembrane protein SMO, resulting in the modulation of the glioma-associated oncogene 1 (GLI1), GLI2 and GLI3, as well as in the processing and binding of GLI activators and repressors to SHH target genes $(5,6)$. Antagonists of SMO have been demonstrated to affect downstream SHH-SMO pathway regulation, and the most clinically advanced SMO targeting agents compete with cyclopamine, an SHH-SMO receptor inhibitor (7). SHH gene deletion mutations in humans have been linked to kidney malformations, such as hydroureter $(8,9)$. Aberrant $\mathrm{SHH}$ signaling is considered to be associated with VACTERL syndrome, which is characterized by renal anomalies (10). In mice, homozygous inactivation of SHH generates a series of defects, including renal aplasia or dysplasia (11). The presence of renal hypoplasia/dysplasia in the ureteric bud lineage of SHH-deficient mice demonstrates a crucial role for $\mathrm{SHH}$ signaling during mammalian renal development (11). However, the mechanisms by which the SHH protein modulates kidney development in mice remain to be elucidated. Therefore, understanding the functional role of the SHH protein in renal 
morphogenesis may provide a novel strategy for preventing and alleviating congenital renal diseases.

Fibroblast growth factors (Fgfs) and their receptors are also known to serve key roles in kidney morphogenesis. A number of Fgf ligands, particularly Fgf2, Fgf7, Fgf8 and Fgf10, are secreted by the mesenchymal and ureteric epithelium in the developing kidney (12-15). Knockout studies in mice have demonstrated the importance of Fgf signaling in the embryonic kidney. Targeted deletion of Fgf7 or Fgf10 $(13,14)$, or their corresponding receptors (Fgfr1 and Fgfr2, respectively) $(16,17)$, results in a reduction in the number of collecting duct branches and loss of the metanephric mesenchyme. The absence of Fgf8 from the metanephric mesenchyme also leads to a decrease in kidney size due to the disruption in nephron formation subsequent to the renal vesicle stage $(12,18)$.

In nonrenal tissues, an increasing number of studies have been investigating the interaction between $\mathrm{SHH}$ and $\mathrm{Fgf}$ proteins during embryonic development (19-22). However, the functional association between $\mathrm{SHH}$ and Fgf proteins in the kidney has not been elucidated. In the present study, the effects of exogenous SHH on Fgf8 and Fgf10 expression levels were investigated using mouse embryonic kidney tissue culture techniques, in order to further understand the regulation of Fgf gene expression during the kidney development.

\section{Materials and methods}

Animal preparation. A total of 28 virgin female BALB/c mouse (obtained from and bred at Biomedical Facility of Shandong University, Jinan, China; 12 h light/dark cycle; temperature, $24^{\circ} \mathrm{C}$; weight, 20-35 g; age, 60 days) were naturally mated overnight and vaginal plugs were identified the following morning. Plug-positive females were transferred to single cages and then sacrificed by cervical dislocation at embryonic day 11.5 (E11.5). Embryonic kidney tissues were obtained between E11.5 and E14.5 and then randomly divided into the control [ 8 female mice; $1 \%$ bovine serum albumin (BSA) in culture medium for 4 days], SHH-treatment (10 female mice; $1.0 \mu \mathrm{g} / \mathrm{ml} \mathrm{SHH}$ protein in culture medium for 4 days) and cyclopamine-treatment groups (10 female mice; $1.0 \mu \mathrm{g} / \mathrm{ml} \mathrm{SHH}$ protein and $10 \mu \mathrm{M}$ cyclopamine in culture medium for 4 days). At least 5 fetuses were obtained from 1 pregnant mouse and 2 embryonic kidneys were obtained from each fetal mouse.

Metanephric explant cultures and surface area measurements. $\mathrm{BALB} / \mathrm{c}$ mouse kidney tissues were obtained from E11.5 embryos using a fine needle syringe and cultured for up to 4 days on polycarbonate filters $(0.45 \mu \mathrm{m}$; EMD Millipore, Billerica, MA, USA) and on simple Trowell culture grids, as described in a previous paper. The tissues were maintained in Dulbecco's modified Eagle's medium-Ham's F12 nutrient mixture, 10\% fetal bovine serum (FBS; Hyclone; GE Healthcare Life Sciences, Chalfont, UK) and penicillin/streptomycin solution (100 U/ml penicillin and $0.1 \mathrm{mg} / \mathrm{ml}$ streptomycin; Beyotime, Institute of Biotechnology, Jiangsu, China). Cyclopamine (cat. no. 239803; EMD Millipore) was dissolved in dimethyl sulfoxide and added to the culture medium at a final concentration of $10 \mu \mathrm{M}$. SHH protein (cat. no. 461-SH; R\&D Systems, Inc., Minneapolis, MN, USA) was dissolved in $1 \%$ BSA (cat. no.
ST023; Beyotime, Institute of Biotechnology, Jiangsu, China) and added to the culture medium at a final concentration of $1 \mu \mathrm{g} / \mathrm{ml}$ for $96 \mathrm{~h}$. The planar surface area was calculated from images of the tissues using the Scion Image version 4.03 software (Scion Co., Frederick, MD, USA), and converted to measurements in $\mathrm{mm}^{2}$ (23). All experimental procedures were performed in accordance with the Guide for the Care and Use of Laboratory Animals (publication no. 85-23, revised 1996; US National Institutes of Health, Bethesda, MD, USA), and were approved by the Institutional Committee for Use and Care of Laboratory Animals of Shandong University (Jinan, China).

Histology and immunohistochemistry (IHC). Mouse embryonic kidney tissue specimens were fixed in $4 \%$ formaldehyde at $4^{\circ} \mathrm{C}$, dehydrated, embedded in paraffin wax and cut into $5-\mu \mathrm{m}$ sections. Following hematoxylin-eosin (HE) staining, tissue sections were analyzed for histological features. For IHC and immunofluorescence analyses, the tissue sections were dewaxed using xylene and rehydrated using a graded ethanol series. Antigens were retrieved by boiling the tissue sections in citrate buffer at $98^{\circ} \mathrm{C}$ for $30 \mathrm{~min}$. The sections were then incubated in $3 \% \mathrm{H}_{2} \mathrm{O}_{2}$ for 30 min to inhibit endogenous peroxidase activity, and blocked with $3 \%$ BSA in phosphate-buffered saline (PBS). Subsequently, sections were incubated overnight at $4^{\circ} \mathrm{C}$ with polyclonal rabbit anti-Fgf8 (dilution, 1:100; cat. no. ABIN1107218; Bioss Inc., Woburn, MA, USA) and polyclonal rabbit anti-Fgf10 (dilution, 1:100; cat. no. ABIN392510; Bioss Inc.) primary antibodies. Goat anti-rabbit antibodies IgG (dilution, 1:1,000; cat. no. ZDR5209; Zhongshanjinqiao, Beijing, China) were used as secondary antibodies. The tetramethylrhodamine (TRITC)-conjugated Dolichos biflorus agglutinin (DBA)-lectin (dilution, 1:2,000; cat. no. L9658; Sigma-Aldrich, St. Louis, MO, USA) was used for immunofluorescence analysis. Nuclei were then counterstained with DAPI.

Calculation of ureteric bud branch points and the number of nephrons. A total of 20 tissue culture explants were embedded in paraffin and sectioned at $5 \mu \mathrm{m}$. Half of the samples were stained with $\mathrm{HE}$ to determine the number of nephrons according to the method described by Hoy et al (24), while others were stained with TRITC-conjugated DBA-lectin to visualize the ureteric buds. The branch points of the nephric duct were then counted in a double-blind study (4 pregnant mice were included in each group).

Microscope and image analysis. Sections of metanephric kidney were visualized and images were captured using a JVC KY-F70 digital camera (JVC, Wayne, NJ, USA) attached to a Leitz DMRB microscope (Leica Microsystems, Wetzlar, Germany), or a Nikon DXM1200 digital camera on a Nikon SMZ1500 stereoscope (Nikon Corp., Tokyo, Japan). Fgf8 and Fgf10 protein expression levels in metanephric explant tissue sections were subjected to microscopic analysis. Briefly, following IHC staining, tissues that were stained purple were selected for analysis. These regions were visualized and staining intensities were quantified using the Image-Pro Plus image analysis software version 7.0 (Media Cybernetics, Inc., Silver Spring, MD, USA). The mean densitometries of the digital images (magnification, $\mathrm{x} 400$ ) were considered to 
represent the Fgf8/Fgf10 staining intensities, and were used to quantify the relative protein expression levels. The staining intensities of tissue areas from 10 randomly-selected fields of view were counted blindly and subjected to statistical analysis.

Reverse transcription-quantitative polymerase chain reaction (RT-qPCR). Mouse embryonic kidneys were harvested between E11.5 and E14.5, and tissues were collected for culturing. Total RNA was extracted using the RNAiso Plus Reagent (cat. no. 9108; Takara Bio, Inc., Tokyo, Japan). A total of 500 ng RNA was reverse transcribed into first strand cDNA using the Primescript RT reagent kit (cat. no. DRR037A; Takara Bio, Inc.). SYBR Premix Ex Taq (10 $\mu$; cat. no. DRR041A; Takara Bio, Inc.) was added to the $\mathrm{qPCR}$ reaction mixture, including $0.4 \mu \mathrm{l}$ forward primer (10 $\mu \mathrm{M}$; Takara Bio Inc.), $0.4 \mu 1$ reverse primer (10 $\mu \mathrm{M}$; Takara Bio Inc.), $2 \mu \mathrm{l} \mathrm{cDNA}$ and $7.2 \mu \mathrm{l}$ distilled $\mathrm{H}_{2} \mathrm{O}$, at a final volume of $20 \mu \mathrm{l}$. The primers used to detect SHH, Fgf8 and Fgf10 mRNA expression levels are listed in Table I. Thermal cycling was performed using the Roche LightCycler 480 system (Roche, Basel, Switzerland). The mRNA expression levels of each target gene were determined using a calibration curve of standards, and expressed relative to GAPDH expression levels.

Western blot analysis. Mouse metanephric kidney tissue samples were homogenized and protein expression levels were determined using western blot analysis, as previously described (7). Briefly, frozen $\left(-80^{\circ} \mathrm{C}\right)$ kidney tissues were washed with $\mathrm{PBS}$ and lysed with radioimmunoprecipitation assay lysis buffer (Beyotime Institute of Biotechnology, Jiangsu, China) containing $50 \mathrm{mM}$ Tris (pH 7.4), $150 \mathrm{mM}$ sodium chloride, $1 \%$ Triton X-100, $1 \%$ sodium deoxycholate, $0.1 \%$ sodium dodecyl sulfate (SDS) and $100 \mathrm{mM}$ phenylmethanesulfonyl fluoride. Protein concentrations were determined using a bicinchoninic acid protein assay (Thermo Fisher Scientific, Inc., Rockford, IL, USA), with BSA as the protein standards. Equal amounts of $50 \mu \mathrm{g}$ total protein were subjected to $12 \%$ SDS-polyacrylamide gel electrophoresis and electrotransferred to polyvinylidene fluoride membranes (EMD Millipore). The membranes were the blocked using 5\% skimmed milk and incubated with primary antibodies at $4{ }^{\circ} \mathrm{C}$ overnight. Subsequently, the membranes were washed three times with Tris-buffered saline and Tween 20 for $10 \mathrm{~min}$, then treated with horseradish peroxidase-conjugated secondary antibodies for $60 \mathrm{~min}$ at $37^{\circ} \mathrm{C}$ and visualized using an electrochemical-luminescence method (Thermo Fisher Scientific, Inc.). Antibodies used for western blot analysis included the following: polyclonal rabbit anti-Fgf8 (dilution, 1:1,000; cat. no. ABIN1107218; Bioss Inc.), polyclonal rabbit anti-Fgf10 (dilution, 1:1,000; cat. no. ab71794; Abcam, Cambridge, MA, USA), mouse monoclonal anti- $\beta$-actin (dilution, 1:5,000; cat. no. A5441; Sigma-Aldrich) primary antibodies, and secondary goat anti-rabbit IgG (dilution, 1:2,000; cat. no. SA00001-2; ProteinTech Group, Inc., Chicago, IL, USA) or goat anti-mouse IgG antibodies (dilution, 1:2,000; cat. no. SA00001-1; ProteinTech Group, Inc.).

Statistical analysis. Statistically significant differences between the experimental and control groups were calculated using the Student's $t$-test or one-way analysis of variance followed

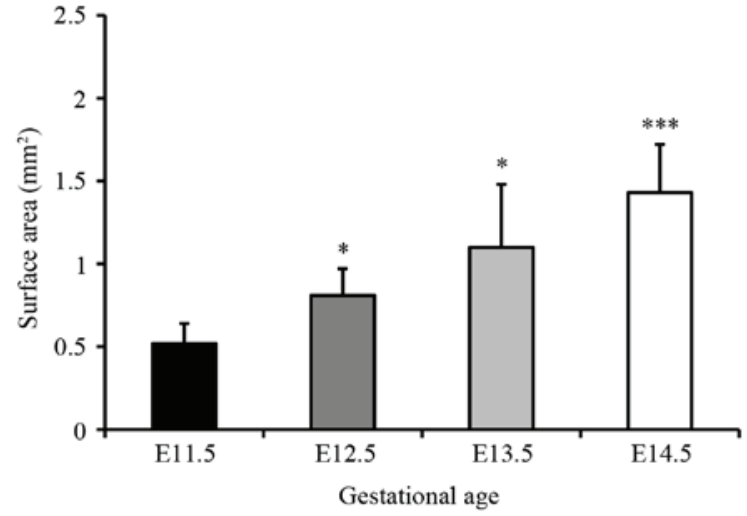

Figure 1. Planar surface areas of BALB/c mouse embryonic metanephric explants at E11.5, E12.5, E13.5 and E14.5 developmental stages. A significant increase in metanephric planar surface areas was observed at more advanced developmental stages indicating the efficient uptake of nutrients from the culture medium. ${ }^{*} \mathrm{P}<0.05$ and ${ }^{* * * *} \mathrm{P}<0.001$ vs. E11.5 group. E, embryonic day.

by pair-wise multiple comparisons with the least-significant difference method using SPSS software (version 13.0; SPSS, Inc., Chicago, IL, USA). $\mathrm{P}<0.05$ was considered to indicate a statistically significant difference.

\section{Results}

Planar surface areas of metanephric explants. Embryonic metanephric explants were obtained under sterile conditions from pregnant BALB/c mice at the E11.5 developmental stage. Between E11.5 and 14.5, the planar surface area of metanephric explants, which is a valid approximation of kidney size, increased in a time-dependent manner ( $\mathrm{P}=0.012$; Fig. 1). The increase in the planar surface area of the explants was used to monitor growth due to its correlation with kidney volume and ureteric bud branching.

Expression levels of SHH, Fgf8 and FgflO mRNA between E11.5 and E14.5. As shown in Fig. 2, the expression levels of SHH, Fgf8 and Fgf10 mRNA in mouse embryonic metanephric explants increased between E11.5 and E14.5, as determined by RT-qPCR. At E11.5, the expression levels of all three genes were relatively low. As demonstrated in Fig. 2A, the expression of SHH increased at E12.5, however, this did not reach statistical significance when compared with other developmental time points ( $\mathrm{P}=0.140$; Fig. 2A). By contrast, at E13.5 and E14.5, SHH expression increased significantly compared with the E11.5 time points $(\mathrm{P}=0.041$ and $\mathrm{P}=0.0008$, respectively; Fig. 2A). Similarly, the expression of Fgf8 increased at E12.5, but with no statistically significant difference observed ( $\mathrm{P}=0.067$; Fig. 2B). By contrast, at E13.5 and E14.5, the expression levels of Fgf8 increased significantly compared with those at E11.5 or E12.5 $(\mathrm{P}=0.032$ and $\mathrm{P}=0.006$, respectively; Fig. 2B). Furthermore, the expression levels of Fgf10 mRNA at E12.5, E13.5 and E14.5 were significantly increased compared with the level at $\mathrm{E} 11.5(\mathrm{P}=0.0008, \mathrm{P}=0.006$ and $\mathrm{P}=0.0009$, respectively; Fig. 2C); however, no significant diference in Fgf10 expression was observed between the E12.5 and E14.5 time points $(\mathrm{P}=0.110$; Fig. 2C). 
Table I. Primer sequences used for polymerase chain reaction.

\begin{tabular}{lll}
\hline Gene & \multicolumn{1}{c}{ Forward $\left(5^{\prime} \rightarrow 3^{\prime}\right)$} & \multicolumn{1}{c}{ Reverse $\left(5^{\prime} \rightarrow 3^{\prime}\right)$} \\
\hline SHH & AGCAGACCGGCTGATGACTC & TCACTCCAGGCCACTGGTTC \\
Fgf8 & CATCAACGCCATGGCAGAA & TCTCCAGCACGATCTCTGTGAATAC \\
Fgf10 & CTGACACATGACCATGGACCAC & TCCAACGTCTGCACTATTTGCTG \\
GAPDH & AAATGGTGAAGGTCGGTGTGAAC & CAACAATCTCCACTTTGCCACTG
\end{tabular}

SHH, sonic hedgehog; Fgf, fibroblast growth factor.
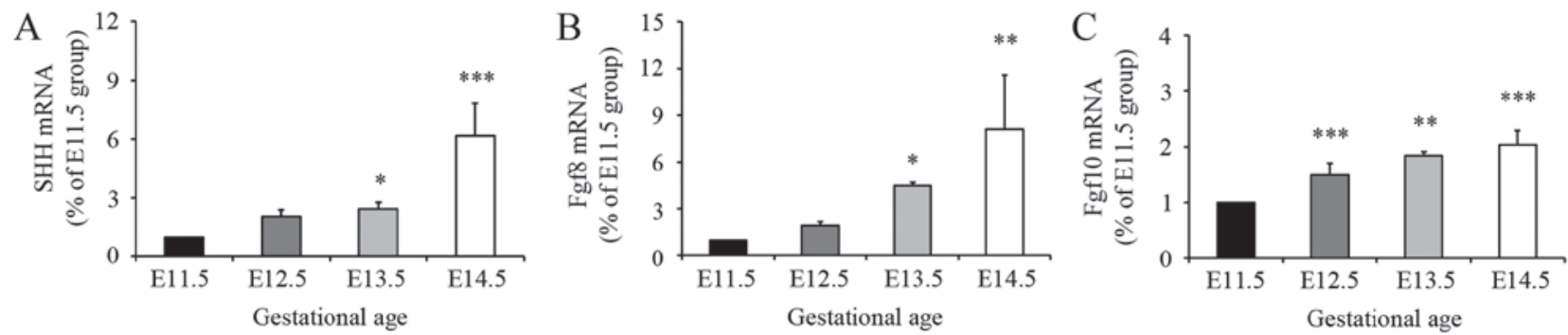

Figure 2. mRNA expression levels of (A) SHH, (B) Fgf8 and (C) Fgf10 in normal BALB/c mouse embryonic kidney tissue explants at E11.5, E12.5, E13.5 and E14.5 gestational ages. For each sample, the mRNA expression levels were normalized to GAPDH mRNA expression ( $\mathrm{n}=7$ for each treatment group). "P<0.05, ${ }^{* *} \mathrm{P}<0.01$ and ${ }^{* * *} \mathrm{P}<0.001$ vs. E11.5 group. SHH, sonic hedgehog; Fgf, fibroblast growth factor; E, embryonic day.

Effect of exogenous SHH on ureteric bud branching morphogenesis and nephrogenesis. Compared with the control tissues, mouse embryonic kidney explants treated with exogenous $\mathrm{SHH}$ protein demonstrated a significant increase in the number of ureteric bud branches (Fig. 3A-C) and the number of nephrons (Fig. 3D-F). The number of formed ureteric bud branch points increased by $25 \%$ following treatment with SHH ( $\mathrm{P}=0.032$; Fig. 3C). In addition, histological analysis of the embryonic kidney sections revealed that $\mathrm{SHH}$ protein treatment significantly increased the number of nephrons by $35 \%(\mathrm{P}=0.022$; Fig. 3F). These results suggest that SHH-SMO signaling serves a crucial role during ureteric bud branching morphogenesis and nephrogenesis.

Effect of exogenous SHH on Fgf8 and FgflO mRNA expression levels. Compared with the control tissues, treatment of embryonic kidney explants with exogenous $\mathrm{SHH}$ protein significantly reduced the Fgf8 mRNA expression by $71 \%$ $(\mathrm{P}=0.007$; Fig. 4A). By contrast, exposure to cyclopamine was associated with a significant increase in Fgf8 mRNA expression by $417 \%$ ( $\mathrm{P}=0.009$; Fig. $4 \mathrm{~A})$ compared with the $\mathrm{SHH}$-group. However, no significant alterations in the expression levels of Fgf10 mRNA were observed following the addition of $\mathrm{SHH}$ protein alone or in combination with cyclopamine $(\mathrm{P}=0.31$ and $\mathrm{P}=0.27$, respectively; Fig. $4 \mathrm{~B}$ ). These results indicate that exogenous SHH protein reduced Fgf8 mRNA expression but had little effect on Fgf10 expression.

Effect of exogenous SHH on Fgf8 and Fgflo protein expression levels. IHC staining demonstrated positive Fhg8 expression primarily in the nephrons and regions of the renal tubules of mouse embryonic kidney tissue explants (Fig. 5A). Compared with control group, the integral optical density (IOD) values of Fgf8 staining decreased by $24 \%$ in the SHH-treated group $(\mathrm{P}=0.028$; Fig. 5B), while the IOD values were increased by $46 \%$ in the $\mathrm{SHH}+$ cyclopamine-treated group $(\mathrm{P}=0.013$; Fig. 5B). In contrast to Fgf8, Fgf10 protein expression was detected primarily in the renal tubules (Fig. 5A). However, no significant difference in the IOD values for Fgf10 was observed between the control and treatment groups (Fig. 5B). Western blot analysis demonstrated that $\mathrm{SHH}$ treatment was associated with a significant reduction in Fgf8 protein expression levels by $40 \%$ compared with the control group $(\mathrm{P}=0.006$; Fig. $5 \mathrm{C}$ and D), whereas the addition of cyclopamine significantly increased the Fgf8 protein expression levels compared with the group treated with $\mathrm{SHH}$ alone ( $\mathrm{P}=0.005$; Fig. $5 \mathrm{C}$ and $\mathrm{D})$. However, no significant alterations in the protein expression levels of Fgf10 were observed in the groups treated with $\mathrm{SHH}$ alone or with $\mathrm{SHH}+$ cyclopamine compared with the control group ( $\mathrm{P}=0.093$; Fig. 5C and $\mathrm{D})$.

\section{Discussion}

In the present study, it was demonstrated that exogneous $\mathrm{SHH}$ protein treatment of mouse embryonic kidney explants was associated with a significant increase in the number of the ureteric bud branches and the number of nephrons. In addition, exogenous SHH protein treatment significantly reduced the Fgf 8 mRNA and protein expression levels, but had no significant effect on the Fgf10 levels. These results demonstrated that $\mathrm{SHH}$ is involved in the modulation of renal morphogenesis and Fgf8 expression in BALB/c mouse metanephric explant cultures, thus suggesting that $\mathrm{SHH}$ is an important developmental regulator of renal morphogenesis.

A previous study has demostrated that kidney development and gene expression patterns in ex vivo organ culture 

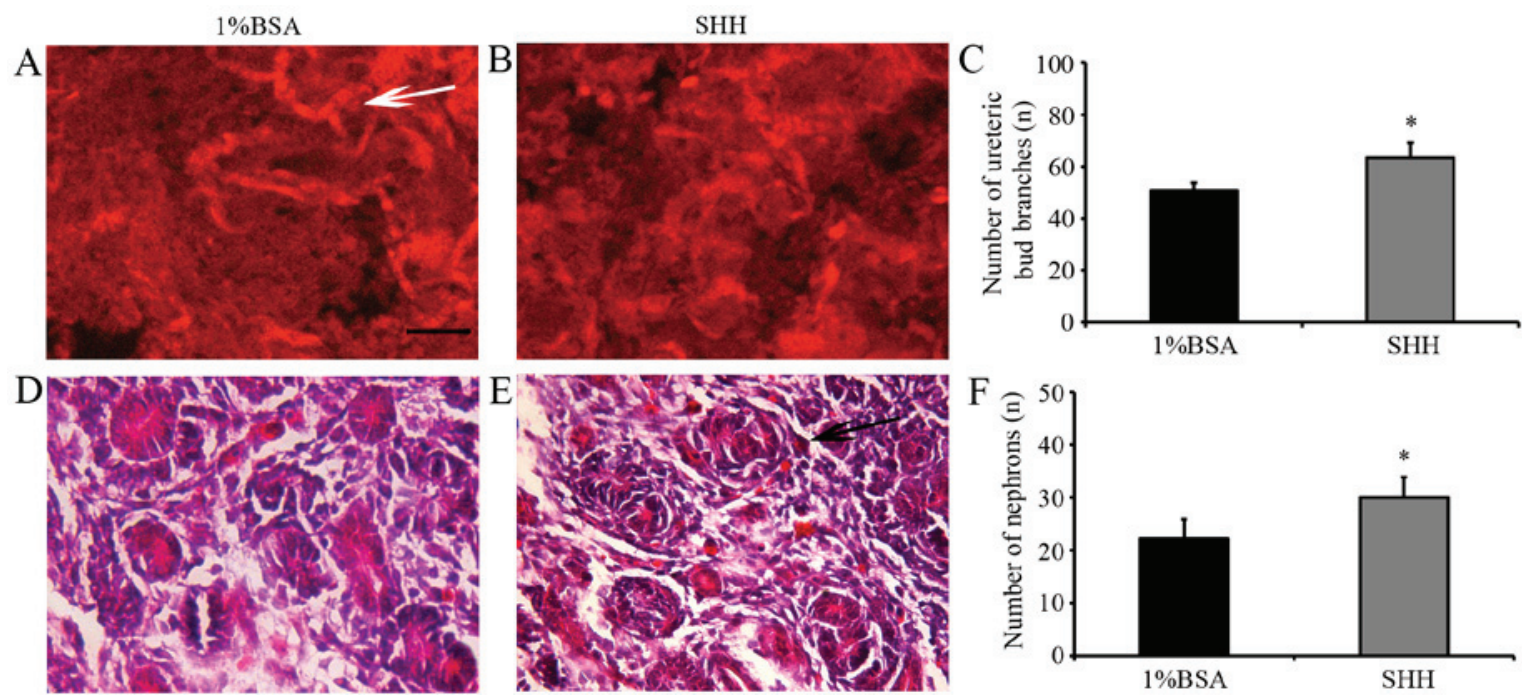

Figure 3. Identification of ureteric bud branches and epithelial metanephric derivatives. DAB staining of BALB/c mouse embryonic tissue explants following 4 days of treatment with (A) $1 \%$ BSA (control) and (B) $1 \%$ BSA + SHH. (C) The number of ureteric bud branches (white arrow) in control and SHH-treated tissues. HE staining of tissue explants following 4 days of treatment with (D) 1\% BSA and (E) 1\% BSA + SHH. (F) The number of nephrons (black arrow) in control and SHH-treated tissues (scale bar, $125 \mu \mathrm{m}$ ). ${ }^{*} \mathrm{P}<0.05$ vs. $1 \%$ BSA-treated (control) group. DAB, Dolichos biflorus agglutininin; BSA, bovine serum albumin; HE, hematoxylin-eosin; SHH, sonic hedgehog.
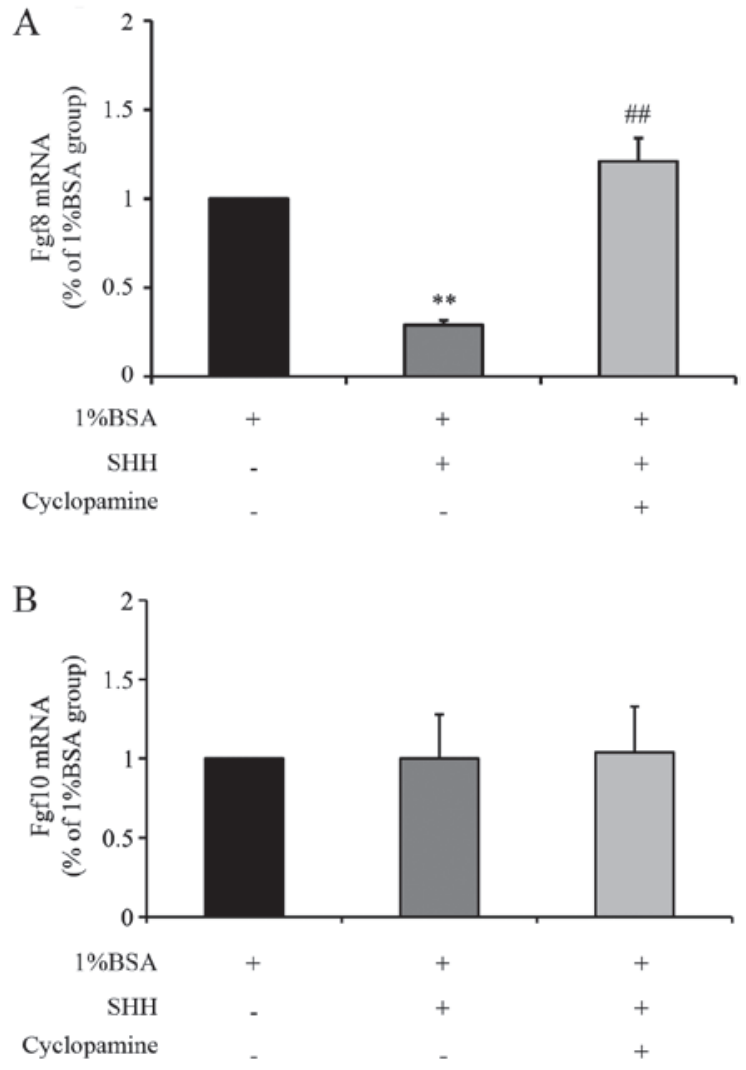

Figure 4. mRNA expression levels of (A) Fgf8 and (B) Fgf10 mRNA levels in $\mathrm{BALB} / \mathrm{c}$ mouse kidney tissue explant cultures following treatment with $1 \%$ BSA, $1 \%$ BSA + SHH and $1 \%$ BSA + SHH + cyclopamine for 4 days. Fgf8 and Fgf10 expression levels were normalized to GAPDH mRNA expression levels ( $\mathrm{n}=7$ for each treatment group). ${ }^{* *} \mathrm{P}<0.01$ vs. $1 \%$ BSA-alone group; ${ }^{\# \#} \mathrm{P}<0.01$ vs. $1 \% \mathrm{BSA}+\mathrm{SHH}$ group. Fgf, fibroblast growth factor; BSA, bovine serum albumin; SHH, sonic hedgehog.

recapitulates early development in vivo (25). The ureteric bud grows and branches as part of a process known as branching morphogenesis, which establishes the collecting duct network of the kidney. A reciprocal signaling network exists between the ureteric bud and the metanephric mesenchymal tissues, which is responsible for establishing the final structure of the kidney and forming the majority of nephrons. Mouse metanephric explant models have been used extensively for the analysis of branching morphogenesis and nephron formation in response to exogenous factors that can be directly added to the culture medium, such as growth factors, agonists or inhibitors $(23,25)$.

SHH is an inhibitory ligand for the PTC receptor, which constituitively inhibits SMO. Following activation by SHH binding, SMO inhibits the processing of full-length GLI3 to a smaller protein product that functions to repress target gene transcription, and stimulates translocation of GLI1 and GLI2 to the nucleus. In the nucleus, GLIs activate transcription of multiple downstream effectors, such as Pax2 and Sall1 (1). These factors are required for nephron development, and loss of their function in the developing metanephric mesenchyme results in renal agenesis or hypoplasia $(26,27)$. According to the results of the present study, SHH gene expression levels were low at the E11.5 and E12.5 developmental stages, and increased at E13.5 and at E14.5. This suggests that SHH serves an important role during the formation of ureteric bud branches and nephrons, but may not be required for the development of mesonephros and its derivatives. This is consistent with the findings of previous in vivo studies $(11,28)$. The number of ureteric bud branches has been demonstrated to affect the number of formed nephrons (2-4). Following addition of SHH protein into the culture, there was an increase in ureteric bud formation, which may have led to the observed increase in nephron formation. Based on the DBA and HE staining results of the present study, the addition of SHH protein to the culture of explanted mouse embryonic kidney tissues increased the number of ureteric bud branches and enhanced the formation of nephrons. Consistent with these observations, the results 
A
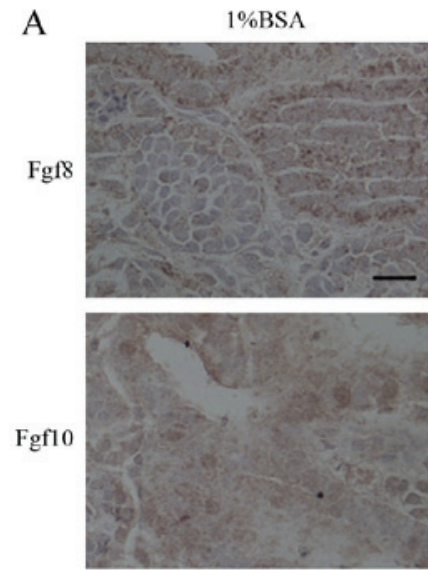

$\mathrm{C}$

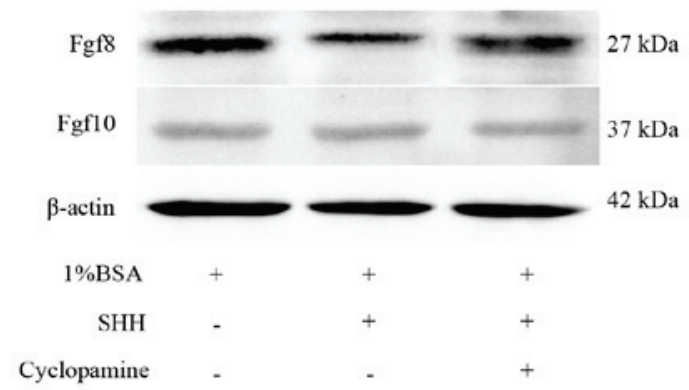

B
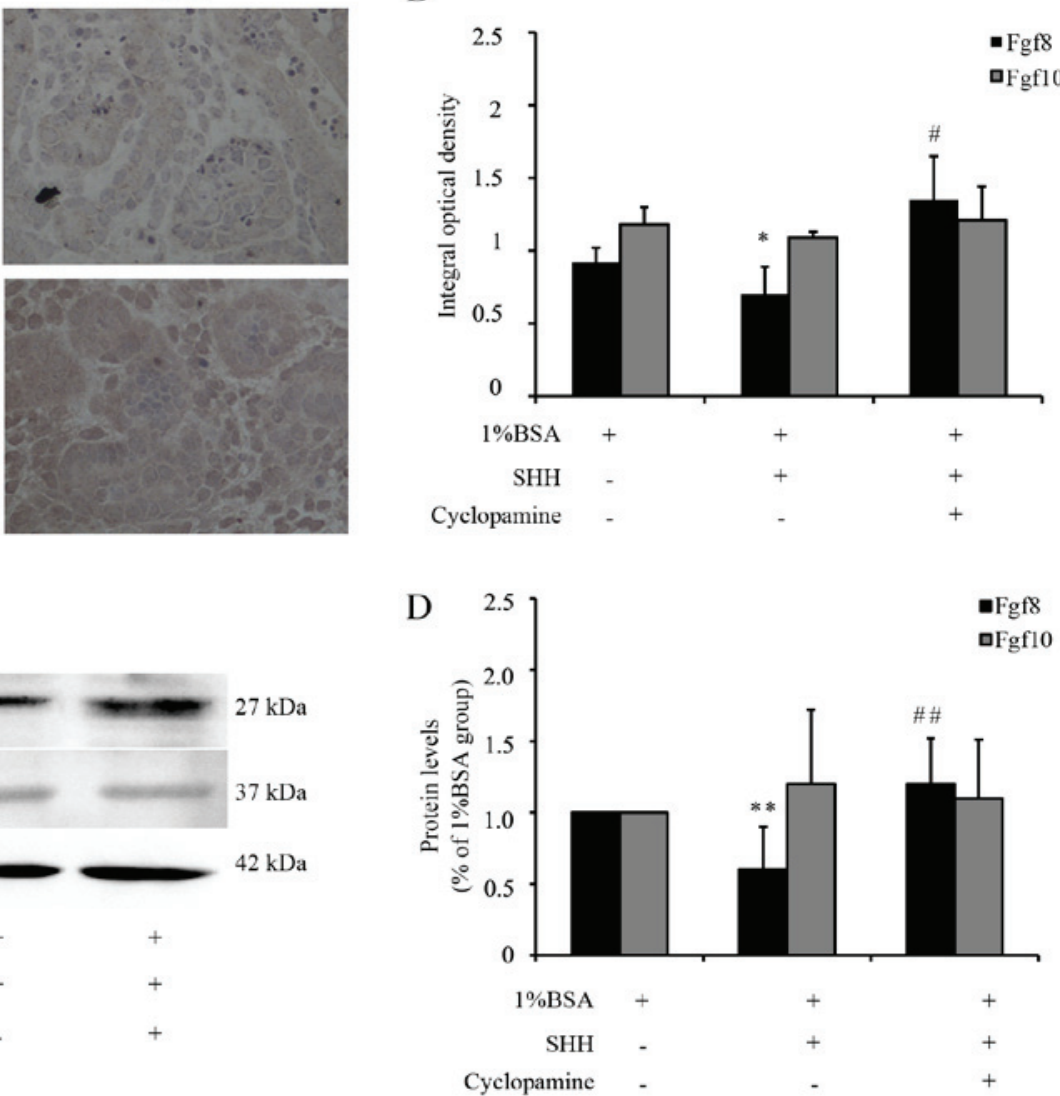

Figure 5. Analysis of Fgf8 and Fgf10 expression in BALB/c mouse kidney tissue explant cultures following treatment with $1 \%$ BSA (control) or $1 \%$ BSA + SHH for 4 days. (A) Immunohistochemical analysis and (B) integral optical densities of Fgf8 and Fgf10 expression levels in the different treatment groups. (C) Western blot analysis and (D) quantification of western blot results, showing the protein expression levels of Fgf8 and Fgf10 expression levels in the various groups. The protein expression levels were normalized to $\beta$-actin ( $\mathrm{n}=7$ for each treatment group). Scale bar $=125 \mu \mathrm{m}$. ${ }^{*} \mathrm{P}<0.05$ and ${ }^{* *} \mathrm{P}<0.01$ vs. $1 \% \mathrm{BSA}$-alone group; ${ }^{\mathrm{P}}<0.05$ and ${ }^{\# \#} \mathrm{P}<0.01$ vs. $1 \% \mathrm{BSA}+\mathrm{SHH}$ group. Fgf, fibroblast growth factor; BSA, bovine serum albumin; $\mathrm{SHH}$, sonic hedgehog.

of the in vitro experiments suggested that enhancing $\mathrm{SHH}$ signaling may promote kidney development.

Fgf8 is expressed in the early metanephric mesenchyme, and is therefore an attractive candidate ligand for regulating nephron development. A previous study demonstrated that Fgf8 promotes renal mesenchymal-epithelial transformation and condensation; however, early kidney development is not dependent on Fgf8 signaling (12). In the absence of Fgf8, mouse embryonic kidney development is normal until the vesicle formation stage (12). According to the results of the present study, there was no significant difference in Fgf8 mRNA expression between E11.5 and E12.5. However, the expression of Fgf8 mRNA increased significantly at E13.5 and E14.5, which is consistent with its established function in nephron formation (12). In a previous study, Fgf8 was demonstrated to be expressed in metanephric mesenchymal tissues around the ureteric bud at E11.5, in the tubule precursor at E12.0, and in the small tubule precursor condensates, comma-shaped and s-shaped bodies, and glomerular epithelial cells at E14.5 (29). Knockout of Fgf8 (Fgf8\%) in mice has been shown to lead to prenatal lethality, and to be associated with the development of small kidneys and abnormal nephron formation (30). By contrast, Fgf10 is widely expressed in mesenchymal and epithelial tissues, which suggests that Fgf10 may function as an epithelial-mesenchymal signaling molecule $(13,31)$. Global targeting of Fgf10 led to perinatal lethality caused by severe dysgenesis/agenesis of the lungs and limbs (32). Furthermore, Fgf10 knockout mice were observed to develop smaller kidneys and fewer collecting ducts (13). As demonstrated in the present study, Fgf10 mRNA expression levels were relatively low at E11.5; however, they increased and remained high between E12.5 and E14.5. Consistent with a previous study (33), this suggests that Fgf10 primarily functions to regulate the formation of ureteric buds but not nephron formation. In addition, during the process of limb induction and heart formation, Fgf8 and Fgf10 have reciprocal regulatory functions, which emphasizes the critical role of Fgf proteins in organ development $(34,35)$. Therefore, the results of the present study provide evidence demonstrating that Fgf8 and Fgf10 serve key functional roles at distinct stages of kidney development.

The effect of exogenous SHH protein on Fgf8 and Fgf10 protein and mRNA expression levels in mouse embryonic kidney tissue explants were investigated in the present study. The culture medium of tissue explants was supplemented with cyclopamine (an SMO receptor inhibitor) to block $\mathrm{SHH}$ signal transduction, and a mouse recombinant $\mathrm{SHH}$ protein to stimulate SHH-SMO signaling. The results demonstrated that treatment with $\mathrm{SHH}$ protein was associated with a significant decrease in Fgf8 mRNA and protein expression 
levels, whereas treatment with $\mathrm{SHH}$ in combination with cyclopamine was associated with no significant alterations in these expression levels compared with the control group. This suggests that $\mathrm{SHH}$ may negatively regulate Fgf8 expression levels, which is consistent with the results presented by Urban et al (36), demonstrating that ectopic SHH treatment inhibited the Fgf8 expression in aquatic larvae. Reduced Fgf8 signals may increase nephron precursor cell apoptosis, thereby decreasing the number of nephrons during the advanced stages of kidney development (36).

In conclusion, the results of the present study suggested that the $\mathrm{SHH}$ protein serves a crucial role in regulating ureteric branching and nephron formation in metanephric explant tissues from BALB/c mice. In addition, SHH may inhibit Fgf8 expression in the developing kidney. This signaling interaction may explain, in part, the regulatory effects of $\mathrm{SHH}$ protein expression on Fgf signaling in vitro (37). The results of the present study also suggest that $\mathrm{SHH}$ may serve an indirect functional role during nephrogenesis, similar to its indirect role during head ectoderm development (21). Despite the fact that the association between $\mathrm{SHH}$ and Fgf signaling in vivo remains unclear, the present study demonstrated the presence of an SHH-Fgf signaling pathway that functions to regulate embryonic kidney development. These observations may provide valuable information for the prevention and therapeutic targeting of congenital kidney malformations.

\section{Acknowledgements}

The present study was financially supported by the Scientific and Technological Projects of Shandong Province (grant no. 2008-01-03-41-01).

\section{References}

1. Gill PS and Rosenblum ND: Control of murine kidney development by sonic hedgehog and its GLI effectors. Cell cycle 5: 1426-1430, 2006.

2. Shah MM, Sampogna RV, Sakurai H, Bush KT and Nigam SK: Branching morphogenesis and kidney disease. Development 131: 1449-1462, 2004.

3. Reidy KJ and Rosenblum ND: Cell and molecular biology of kidney development. Semin Nephrol 29: 321-337, 2009.

4. Piscione TD and Rosenblum ND: The molecular control of renal branching morphogenesis: Current knowledge and emerging insights. Differentiation 70: 227-246, 2002.

5. Bai CB, Auerbach W, Lee JS, Stephen D and Joyner AL: Gli2, but not Gli1, is required for initial Shh signaling and ectopic activation of the Shh pathway. Development 129: 4753-4761, 2002.

6. Park HL, Bai C, Platt KA, Matise MP, Beeghly A, Hui CC, Nakashima M and Joyner AL: Mouse Gli1 mutants are viable but have defects in SHH signaling in combination with a Gli2 mutation. Development 127: 1593-1605, 2000.

7. Ding H, Zhou D, Hao S, Zhou L, He W, Nie J, Hou FF and Liu Y: Sonic hedgehog signaling mediates epithelial-mesenchymal communication and promotes renal fibrosis. J Am Soc Nephrol 23: 801-813, 2012.

8. Lurie IW, Ilyina HG, Podleschuk LV, Gorelik LB and Zaletajev DV: Chromosome 7 abnormalities in parents of children with holoprosencephaly and hydronephrosis. Am J Med Genet 35: 286-288, 1990.

9. Nowaczyk MJ, Huggins MJ, Tomkins DJ, Rossi E, Ramsay JA, Woulfe J, Scherer SW and Belloni E: Holoprosencephaly, sacral anomalies, and situs ambiguus in an infant with partial monosomy 7q/trisomy $2 \mathrm{p}$ and SHH and HLXB9 haploinsufficiency. Clin Genet 57: 388-393, 2000.

10. Kim PC, Mo R and Hui Cc C: Murine models of VACTERL syndrome: Role of sonic hedgehog signaling pathway. J Pediatr Surg 36: 381-384, 2001.
11. Yu J, Carroll TJ and McMahon AP: Sonic hedgehog regulates proliferation and differentiation of mesenchymal cells in the mouse metanephric kidney. Development 129: 5301-5312, 2002.

12. Grieshammer U, Cebrián C, Ilagan R, Meyers E, Herzlinger D and Martin GR: FGF8 is required for cell survival at distinct stages of nephrogenesis and for regulation of gene expression in nascent nephrons. Development 132: 3847-3857, 2005.

13. Ohuchi H, Hori Y, Yamasaki M, Harada H, Sekine K, Kato S and Itoh N: FGF10 acts as a major ligand for FGF receptor 2 IIIb in mouse multi-organ development. Biochem Biophys Res Commun 277: 643-649, 2000.

14. Qiao J, Uzzo R, Obara-Ishihara T, Degenstein L, Fuchs E and Herzlinger D: FGF-7 modulates ureteric bud growth and nephron number in the developing kidney. Development 126: 547-554, 1999.

15. Barasch J, Qiao J, McWilliams G, Chen D, Oliver JA and Herzlinger D: Ureteric bud cells secrete multiple factors, including bFGF, which rescue renal progenitors from apoptosis. Am J Physiol 273: F757-F767, 1997.

16. Poladia DP, Kish K, Kutay B, Hains D, Kegg H, Zhao H and Bates CM: Role of fibroblast growth factor receptors 1 and 2 in the metanephric mesenchyme. Dev Biol 291: 325-339, 2006.

17. Zhao H, Kegg H, Grady S, Truong HT, Robinson ML, Baum M and Bates CM: Role of fibroblast growth factor receptors 1 and 2 in the ureteric bud. Dev Biol 276: 403-415, 2004.

18. Perantoni AO, Timofeeva O, Naillat F, Richman C, Pajni-Underwood S, Wilson C, Vainio S, Dove LF and Lewandoski M: Inactivation of FGF8 in early mesoderm reveals an essential role in kidney development. Development 132: 3859-3871, 2005

19. White AC, Xu J, Yin Y, Smith C, Schmid G and Ornitz DM: FGF9 and SHH signaling coordinate lung growth and development through regulation of distinct mesenchymal domains. Development 133: 1507-1517, 2006.

20. Zhang Z, Verheyden JM, Hassell JA and Sun X: FGF-regulated Etv genes are essential for repressing Shh expression in mouse limb buds. Dev Cell 16: 607-613, 2009.

21. Mukhopadhyay A, Krishnaswami SR, Cowing-Zitron C, Hung NJ, Reilly-Rhoten H, Burns J and Yu BD: Negative regulation of Shh levels by Kras and Fgfr2 during hair follicle development. Devel Biol 373: 373-382, 2013.

22. Haworth KE, Wilson JM, Grevellec A, Cobourne MT, Healy C, Helms JA, Sharpe PT and Tucker AS: Sonic hedgehog in the pharyngeal endoderm controls arch pattern via regulation of Fgf8 in head ectoderm. Dev Biol 303: 244-258, 2007.

23. Gupta IR, Lapointe M and Yu OH: Morphogenesis during mouse embryonic kidney explant culture. Kidney Int 63: 365-376, 2003.

24. Hoy WE, Douglas-Denton RN, Hughson MD, Cass A, Johnson K and Bertram JF: A stereological study of glomerular number and volume: Preliminary findings in a multiracial study of kidneys at autopsy. Kidney Int Suppl: S31-S37, 2003.

25. Barak H and Boyle SC: Organ culture and immunostaining of mouse embryonic kidneys. Cold Spring Harb Protoc 2011: pdb. prot5558, 2011.

26. Narlis M, Grote D, Gaitan Y, Boualia SK and Bouchard M: Pax2 and pax8 regulate branching morphogenesis and nephron differentiation in the developing kidney. J Am Soc Nephrol 18: 1121-1129, 2007.

27. Chai L, Yang J, Di C, Cui W, Kawakami K, Lai R and Ma Y: Transcriptional activation of the SALL1 by the human SIX1 homeodomain during kidney development. J Biol Chem 281: 18918-18926, 2006.

28. Murashima A, Akita H, Okazawa M, Kishigami S, Nakagata N, Nishinakamura R and Yamada G: Midline-derived Shh regulates mesonephric tubule formation through the paraxial mesoderm. Dev Biol 386: 216-226, 2014.

29. Pedersen A, Skjong C and Shawlot W: Lim 1 is required for nephric duct extension and ureteric bud morphogenesis. Dev Biol 288: 571-581, 2005.

30. Sun X, Meyers EN, Lewandoski M and Martin GR: Targeted disruption of Fgf8 causes failure of cell migration in the gastrulating mouse embryo. Genes Dev 13: 1834-1846, 1999.

31. Igarashi M, Finch PW and Aaronson SA: Characterization of recombinant human fibroblast growth factor (FGF)-10 reveals functional similarities with keratinocyte growth factor (FGF-7). J Biol Chem 273: 13230-13235, 1998.

32. Sekine K, Ohuchi H, Fujiwara M, Yamasaki M, Yoshizawa T, Sato T, Yagishita N, Matsui D, Koga Y, Itoh N and Kato S: Fgf10 is essential for limb and lung formation. Nat Genet 21: 138-141, 1999. 
33. Michos O, Cebrian C, Hyink D, Grieshammer U, Williams L, D'Agati V, Licht JD, Martin GR and Costantini F: Kidney development in the absence of Gdnf and Spryl requires Fgf10. PLoS Genet 6: e1000809, 2010.

34. Xu X, Weinstein M,Li C, Naski M, Cohen RI, Ornitz DM, Leder P and Deng C: Fibroblast growth factor receptor 2(FGFR2)-mediated reciprocal regulation loop between FGF8 and FGF10 is essential for limb induction. Development 125: 753-765, 1998.

35. Watanabe Y, Miyagawa-Tomita S, Vincent SD, Kelly RG, Moon AM and Buckingham ME: Role of mesodermal FGF8 and FGF10 overlaps in the development of the arterial pole of the heart and pharyngeal arch arteries. Circ Res 106: 495-503, 2010.
36. Urban AE, Zhou X, Ungos JM, Raible DW, Altmann CR and Vize PD: FGF is essential for both condensation and mesenchymal-epithelial transition stages of pronephric kidney tubule development. Dev Biol 297: 103-117, 2006.

37. Guo W, Yi X, Ren F, Liu L, Wu S and Yang J: Activation of SHH signaling pathway promotes vasculogenesis in post-myocardial ischemic-reperfusion injury. Int J Clin Exp Pathol 8: 12464-12472, 2015. 